\title{
Digital Chest Radiography: Clinical Aspects
}

\author{
Ulf Tylén
}

Technical development puts the completely digital radiology department within reach. Presently available systems have slightly inferior spatial resolution than film/screen systems. This is well compensated for by image processing. In the future, the digital radiology department working environment will be different. Systems should be designed that conform as much as possible to radiologists' present way of working to facilitate transition to the new system and avoid unnecessary stress.

Copyright $\odot 1995$ by W.B. Saunders Company

KEY WORDS: digital radiography, chest, workstation.

C OMPUTERS are a prerequisite for image creation and image processing in modern radiology. The development of digital techniques in recent years has also led to the use of computers for image archiving and communication. This brings the fully digital radiology department within reach. In such a department, no film would be produced, the images would be sent across a network to diagnostic workstations for interpretation and reporting, and then digitally archived. Images could also be distributed to referring clinicians across the network in operating rooms, wards, or outpatient areas. Although partially digital departments exist in many places in the world, only a few completely digitized departments are functioning in Europe (eg, in Vienna, Austria and Viborg, Denmark).

There are many reasons favoring the department of a digital radiology department. Film is an imperfect detector for $\mathrm{x}$-rays and has a very limited dynamic range. Films tend to be unavailable when you need them and film archives demand considerable space and personnel. Film costs are high, particularly when using laser cameras, and chemicals used for film processing are polluting our waters. Digital techniques permit acquisition of a larger amount of information and comprehension of this information may be increased further by image processing. Picture archiving and communication systems (PACS) also facilitate exchange of information within the radiology department and with referring clinicians. However, there are also problems with the digital technique. The equipment is expensive, a problem which becomes acute if you want to transform an entire department from conventional to modern digital technique. The transition also means that the radiologists and the personnel have to work in a new way, and the environment will change considerably. Handling of more and new information including the need for image processing may be time consuming. Another controversy is whether the limited spatial resolution inherent in the currently available digital imaging systems will hamper the diagnostic work. Today, more than $70 \%$ of all images in a modern radiology department are generated digitally, but less than $20 \%$ of all examinations are performed with digital equipment because the majority of all examinations involve the chest or bones and joints. Therefore, to accomplish the fully digital radiology department, it is necessary to develop acceptable techniques for examination of these organs.

To introduce a new examination technique, at least one of the following conditions must be fulfilled: new diagnostic information is provided; new equipment is easier to work with than the equipment previously used; the new method is more cost efficient.

When, for example, computed tomography (CT) was introduced, all three conditions were fulfilled, and therefore, the method was rapidly accepted. Digital chest radiography faces the same demand; however, it involves special problems. The thorax is a very large object containing several important anatomic structures, yet we demand information about all those structures with a single exposure! We need to handle the huge attenuation differences between the mediastinum and the lung parenchyma, and we also need high spatial resolution to evaluate small details. With conventional radiographic technique, a large amount of scattered radiation is induced. Structural noise caused by superim-

From the Department of Diagnostic Radiology, Sahlgrenska University Hospital, Göteborg, Sweden.

Address reprint requests to Ulf Tylén, $M D, P h D$, Göteborg University, Department of Diagnostic Radiology, Sahlgrenska University Hospital, S-413 45 Göteborg, Sweden.

Copyright $\mathbb{0} 1995$ by W.B. Saunders Company 0897-1889/95/0801-1005\$3.00/0 
position of soft tissues and bones degrades the images further. Conventionally, we try to manage these problems using grids, latitude films, dodgers, asymmetric film/screen combinations, etc, and we consider our results satisfactory. The challenge for digital radiography is to match those results or, rather, to improve them.

\section{TECHNIQUES FOR DIGITAL CHEST RADIOGRAPHY}

Digital chest radiography may be performed with two types of equipment using beamdetector and area-detector techniques. With the former technique, a well-collimated $x$-ray beam in the shape of a fan or a pencil scans through the object. This technique, called scanned projection radiography, uses detectors of high efficiency, and scattered radiation is markedly reduced because of the collimation of the beam. This means that the radiation dose to the patient may be reduced. However, the equipment used is complex and expensive and the scanning demands an exposure time of several seconds, putting stress on the $\mathrm{x}$-ray tube. So far, equipment for scanned projection radiography of the chest has been experimental. Image intensifiers, storage-phosphor plates and selenium drums are used as area detectors in various commercially available equipment for digital chest radiography. However, the basic problems of film/screen radiography remain: the need for a large dynamic range, low inherent contrast because of scattered radiation and structural noise, as well as the need for high spatial resolution to evaluate small details.

\section{EVALUATION OF DIAGNOSTIC PERFORMANCE}

Evaluation of a new radiographic technique is usually based on physical measurements of parameters such as spatial resolution, contrast, image noise, etc. However, the system that measures best physically does not always perform best in the clinical situation. ${ }^{1}$ Therefore, evaluation should include diagnostic tests involving the psycho physical process that allows the radiologist to detect a lesion, recognize the size, shape, etc, and interpret it as a disease entity.

\section{Physical Measurement}

Among physical parameters, the limited spatial resolution of digital chest radiography sys- tems seems to be the most controversial. A conventional film/screen system has a spatial resolution of at least $6 \mathrm{lp} / \mathrm{mm}$ corresponding to a pixel size of $0.08 \mathrm{~mm}$. However, the measurements are made with phantoms providing $100 \%$ contrast. Examination of a patient introduces a significant amount of scattered radiation that reduces the contrast to $1 \%$ to $3 \% .^{1,2}$ This means that the spatial resolution decreases to about 2 $\mathrm{lp} / \mathrm{mm}$ corresponding to a pixel size of $0.25 \mathrm{~mm}$. In digital radiography, the pixel size determines the spatial resolution. Pixel size is determined by the field of view (FOV) and the image matrix. For digital chest radiography the FOV is typically $40 \mathrm{~cm}$ and the image matrix $2,048 \times$ 2,048 pixels $(2 \mathrm{~K})$ for storage-phosphor systems and $1,024 \times 1,024$ pixels $(1 \mathrm{~K})$ for image intensifiers. This gives a pixel size of 0.2 and 0.4 $\mathrm{mm}$, respectively. Thus, the difference in spatial resolution between the two systems is small. As long as the image contrast cannot be improved, the demand for high spatial resolution has probably been exaggerated. Instead all effects should be made to improve image contrast, eg, by use of scanned projection radiography. Image contrast may also be improved by image processing. This has been shown in systems using a large image intensifier ${ }^{3,4}$ as well as those with storage-phosphor plates. 5,6 It is only the image contrast that is increased. Image processing may even introduce artifacts.

\section{Diagnostic Performance}

The need for diagnostic accuracy depends on the clinical situation. A significant number of all chest radiographs are obtained using portable techniques for patients in intensive care unit. In this situation, it is very difficult to obtain highquality chest radiographs even with film/screen systems because of a lack of automatic exposure control and the difficulties in positioning the patient. If no grid is used, there is also a very high contribution of scattered radiation. However, the referring clinician does not require diagnosis of subtle interstitial disease, but rather wants to know the position of indwelling lines and tubes and whether pneumothorax developed during intubation. He also wants to know there is a significant amount of pulmonary edema or pleural effusion and possibly whether there are significant atelectases. In this setting, 
the only digital technique available is storagephosphor plates. Many reports comparing film/ screen techniques and storage-phosphor plates have proven that the latter permit better overall diagnosis of most conditions because of improved image quality. ${ }^{7}$ Storage-phosphor plates also permit better diagnosis of the position of cardiovascular devices. ${ }^{1}$ However, in the diagnosis of a small pneumothorax, some controversy remains whether phosphor plates are adequate. ${ }^{8}$ The main problem concerning diagnosis of pneumothorax is that only about a third of these are diagnostic yield. ${ }^{9}$ Examination in a lateral or lateral decubitus projection improves the diagnostic yield, but in a difficult clinical situation, CT is the method of choice for diagnosing small loculated pneumothorax. ${ }^{9,10}$

In the routine clinical situation, upright anteroposterior and lateral views may be obtained. Investigations using anthropomorphic phantom as well as clinical patients have shown that with the storage phosphor plate technique and image processing, the existing small differences in spatial resolution are well compensated for. The plates perform well in nodule detection 5,6 as well as in diagnosis of interstitial disease. ${ }^{11,12}$ However, very subtle interstitial changes may be difficult to show, but with the recently developed phosphor plates with a pixel size of 0.1 $\mathrm{mm}$, even subtle changes may be diagnosed. However, in the clinical situation, mere demonstration of lesions is not sufficient. Lesions must also be interpreted as to pattern, localization and extension to suggest a disease entity. This is done better with CT and particularly with highresolution CT (HRCT). In good clinical practice, the patient with clinical symptoms in whom interstitial disease, eg, may not be demonstrated by chest radiography should be examined by HRCT. In the same way, the patient in whom subtle interstitial changes are demonstrated should be examined by CT scan to obtain more information on the localization and character of the lesions. CT, or more precisely HRCT, is the method of choice for evaluating parenchymal lung disease. Therefore, the role of chest radiography with conventional or digital technique will become less important in the future. This is supported by the fact that reading chest radiographs is a complicated task. It is well known that a large number of parenchymal lesions are recognized only at the second or third examination when they have grown significantly. The reason for this is often that the radiologist does not recognize the lesion because of either poor knowledge, inexperience, or stress in the reporting situation. We also have to deal with a very high intrareader and interreader variation when interpreting the findings. Considering these aspects, the presently used matrix of $2 \mathrm{~K}$ corresponding to a pixel size of 0.2 $\mathrm{mm}$ will be completely sufficient for routine clinical work. When contemplating investment of more money into a digital chest radiography system with higher resolution, it may be better to invest the money in double reading, education of the radiologists, or a better working environment.

\section{WORKSTATIONS}

Films will disappear in the completely digital radiology department of the future. Examinations will be performed using storage-phosphor plates, scanning devices, or other types of digital equipment permitting direct digitization and transfer of the image information across a network to the workstation. Technicians who have experienced digital radiology usually accept the new technique immediately, and high and uniform image quality, few retakes, and less cassette handling are some immediate advantages. Several reports claim that the diagnostic result from reading images on monitors is equivalent to hard-copy reading. ${ }^{13-15}$ However, the new environment for interpreting images and reporting may be stress inducing for the radiologists, and it has been reported that the time for reading is longer than when using conventional film. ${ }^{16}$ This must be obviated or radiologists will not accept the new working environment. To encourage monitor reading among more conservative chest radiologists, the software of the workstation must be written so that it mimics the situation of reading film from a light box as much as possible. This demands analysis of the present working conditions as much as future possibilities. Today most radiologists interpret and report from light boxes. Thus, he has access to information from the newly acquired film and, usually, film from previous examinations. In addition, he has information from the referring physician's request and reports of the 
radiographic findings in the previous examinations. All this information is brought to the light boxes with the film hung by technicians, secretaries, or other personnel. When the radiologist has finished reporting on the patient, he just presses a button and up comes the next. A similar work situation must be developed for diagnoses performed in front of a monitor. Images and information from the request and previous reports must be prepared by the digital system using prefetching according to a work list provided by the radiologic information system (RIS). This demands thorough integration of the RIS with the PACS. To design such a system for chest radiography, we are presently working with a concept of two monitors containing image information and one monitor containing patient data including information from the request and previous reports. When the radiologist starts his work, he only clicks one button labeled "next patient" with his mouse. Immediately, the anteroposterior and lateral images of the examination to be reported are displayed on the two monitors and the appropriate text information is displayed on the text monitor. The images have a correct orientation and are windowed according to preset parameters. With a single mouse click on an easily found button, the image combination may be changed to display the new and most recent AP or lateral images. It is also possible to recall a particular other image combination by finding the appropriate report and clicking the button. Under these or similar conditions, the radiologist may recognize his present working conditions in the new system and more readily accept the new environment. We also know that image processing is time consuming and think that there will be time only for simple windowing and zooming. The zoom function will be particularly useful in situations where reporting is done from $1-\mathrm{K}$ monitors when the images were acquired using a $2-\mathrm{K}$ system.

The transition to an all-digital radiography department is a complicated task. There is no question that digital image quality is sufficient for diagnostic work. However, every effort must be made to facilitate the radiologist's working situation as much as possible. Only then will the introduction of new techniques be accepted by the majority of radiologists. Clinicians must provide the money by referring patients to the all-digital radiology department. Therefore, advantages in clinical care must also be provided to persuade clinicians to use the system. A PACS permits image distribution across the network to workstations or monitors all over the hospital. These images may be distributed immediately after an examination is complete, which would be very desirable from a clinical point of view. However, from the radiologists point of view, images must not be released across the hospital network unless accompanied by the radiologist's report. The system must be designed so that when images are displayed on the clinician's monitor, the radiologist's report simultaneously appears. This may quite easily be accomplished by integration of the PACS, RIS, and the hospital information system. However, because the digital images may be distributed almost immediately across the network, radiologists must immediately report findings during weekends and night calls as well. Teleradiology may be helpful in this situation.

Techniques for digital chest radiography are available today. The process of transition to the all digital environment demands thorough analysis of the present working conditions. The new system must be designed so that we will not be mastered by it. It must be so flexible that a radiologist's individual working habits are not affected. Only radiologists themselves can design such a system.

\section{REFERENCES}

1. Niklason LT, Chan HP, Cascade PN, et al: Portable chest imaging: Comparison of storage phosphor digital, asymmetric screen-film, and conventional screen-film systems. Radiology 186:387-393, 1993

2. Mânsson LG, Kheddache S, Schlossman D, et al: Digital chest radiography with a large image intensifierEvaluation of diagnostic performance and patient exposure. Acta Radiol 30:337-342, 1989

3. Angelhed JE, Månsson LG, Kheddache S: SPIE Proc 1444:159-170, 1991
4. Kheddache S, Månsson LG, Angelhed JE, et al: Effects of optimization and image processing in digital chest radiography. An ROC study with an anthropomorphic phantom. Eur J Radiol 13:143-150, 1991

5. Schaefer CM, Greene R, Llewellyn HJ, et al: Interstitial lung disease: Impact of post-processing in digital storage phosphor imaging. Radiology 178:733-738, 1991

6. Procop M, Schaefer CM, Oestmann J, et al: Improved parameters for unsharp mask filtering of digital chest radiographs. Radiology 187:521-526, 1993 
7. Wandtke JC: Bedside chest radiography. Radiology 190:1-10, 1994

8. Elam EA, Rehm K, Hillman BJ, et al: AJR 158:509514,1992

9. Carr JJ, Reed JC, Choplin RH, et al: Plain and computed radiography for detecting experimentally induced pneumothorax in cadavers: Implications for detection in patients. Radiology 183:193-199, 1992

10. Morgan RA, Owens CM, Collins CD, et al: Detection of pneumothorax with lateral shoot-through digital radiography. Clin Radiol 48:249-252, 1993

11. Kehler M, Albrechtsson U, Andrésdóttir A, et al: Digital luminescence radiography in interstitial disease. Acta Radiol 32:18-23, 1991

12. Kido S, Ikezoe J, Takeuchi N, et al: Interpretation of subtle interstitial lung abnormalities. Conventional versus storage phosphor radiography. Radiology 187:527-533, 1993

13. Slasky BS, Gur D, Good WF, et al: Receiver operating characteristic analysis of chest image interpretation with conventional, laser-printed, and high-resolution workstation images. Radiology 174:775-780, 1990

14. Lyttkens K, Andersson B, Kehler M, et al: Bedside chest radiography using digital luminescence. A comparison between digital radiographs reviewed on a personal computer and as hard-copies. Acta Radiol 33:427-430, 1992

15. Beard DV, Hemminger BM, Perry JR, et al: Interpretation of CT studies: Single-screen workstation versus film alternator. Radiology 187:565-569, 1993

16. Razawi M, Sayre JW, Taira RK, et al: Receiveroperating-characteristic study of chest radiographs in children: Digital hard-copy film vs $2 \mathrm{k} \times 2 \mathrm{k}$ soft-copy images. AJR 158:443-448, 1992 